ORIGINAL ARTICLE

\title{
Suspicious urinary cytology with negative evaluation for malignancy in the diagnostic investigation of haematuria: how to follow up?
}

G Nabi, D Greene, M O Donnel

J Clin Pathol 2004;57:365-368. doi: 10.1136/jcp.2003.009696

See end of article for authors' affiliations

Correspondence to: Mr G Nabi, Department of Surgery, Medical School, Polwarth Building,

University of Aberdeen, Aberdeen AB25 2ZD, UK; g.nabi@abdn.ac.uk

Accepted for publication 29 June 2003
Aims: To define the natural history of patients with suspicious urinary cytology and negative initial evaluation for malignancy in the investigation of haematuria.

Patients and methods: Data from the hospital information support system on urinary cytology examinations carried out at one centre were audited over a period of 24 months. There were 102 patients who had suspicious urinary cytology for malignant cells with negative initial evaluation. Follow up investigations, treatment, and final outcome were noted.

Results: There were 102 patients with suspicious urinary cytology and negative initial evaluation for malignancy in 24 months, with a mean follow up of 15.7 months. Seventy patients had no obvious pathology on initial investigations. Forty one patients were found to have urological malignancies (29 bladder, eight ureteric, and four prostate) on follow up. All patients diagnosed as having urothelial malignancies on follow up had either persistent suspicious cytology (29) or recurrent haematuria (eight). The mean duration for appearance of lesions was 5.6 months (range, 3-12 months). Three patients had suspicious digital rectal examination and biopsies confirmed adenocarcinoma of the prostate. One patient had urinary retention and transurethral resection of prostate showed prostatic adenocarcinoma. The presence of suspicious cells on repeat urine analysis was the only significant factor in predicting the presence of urothelial tumours ( $p=0.002$ ).

Conclusion: Patients with persistent suspicious/positive cytology or recurrent haematuria need further evaluation and follow up. Asymptomatic patients or patients with obvious benign pathology do not require repeat evaluation. Careful urological evaluation, including prostate, should be carried out in these patients.
$\mathrm{T}$ he widely used Papanicolaou and Marshal $^{1}$ classification of urinary cytological examination considers class I-II as negative and class IV-V as positive for the presence of malignant cells. The class III or suspicious group remains controversial. Suspicious urinary cytology in clinical practice necessitates thorough investigations to rule out the presence of urothelial malignancy. Most clinicians treat this as malignant, leading to repeated evaluations and follow up, which in most of these patients remain inconclusive. Lack of guidelines in the literature regarding the management of these patients, variations in urine specimen processing, subjective interpretation of cytological analysis, and lack of a standard method of reporting urinary cytology are some of the difficulties in decision making on this issue. Clearly, there remains a group of patients who are over investigated. We analysed our results in the present study to answer the question of how to follow up suspicious urinary cytology in a patient with a history of haematuria and normal initial investigations? We defined suspicious urinary cytology as (a) not clearly indicative of malignancy or (b) cannot clearly exclude the presence of malignancy. The aims of our study were:

(1) To define the natural history of patients with suspicious urine cytology and negative evaluation for malignancy.

(2) To determine a subgroup of these patients with increased risk of developing malignancy.

(3) To develop guidelines for the follow up of these patients.

\section{PATIENTS AND METHODS}

Data from the hospital information support system on urinary cytology examinations carried out at one centre were audited over a period of 24 months. Age, sex, history of persistent microscopic or gross haematuria, history of smoking, occupational exposure, follow up investigations, treatment, and final outcome were noted from the hospital records. A record of clinical profile and investigations generated by suspicious urinary cytology was made. Patients with obvious malignancy on initial investigations were excluded from our study. A group of patients who had suspicious urinary cytology (not clearly malignant or cannot exclude presence of malignancy) with negative initial evaluation was defined. The initial investigations usually comprised flexible cystoscopy, intravenous urogram, and abdomenal ultrasound, followed by rigid cystoscopy with bilateral ureteric washings and retrograde studies, wherever necessary. If washings or retrograde studies showed an abnormality, ureteroscopy (with or without biopsies) was carried out. Patients with negative initial investigations were followed up in clinic after six to eight weeks and repeat urinary cytology was requested. Persistence of suspicious urinary cytology or symptoms required repeat evaluation. Patients in whom repeat urinary cytology at six to eight weeks showed no evidence of malignant cells were followed at three monthly intervals, both clinically and with repeat voided urinary cytological analysis.

\section{RESULTS}

There were 102 patients with suspicious urinary cytology and negative initial evaluation for malignancy among those in 
whom cytological examinations were carried out over a period of 24 months. The mean follow up of our study was 15.7 months (range, 10-24). There were 76 male and 26 female patients. The mean age was 61.7 (range, 51-72) years. A history of smoking was present in 60 patients. Patients were divided into two groups (figs 1 and 2). Group 1 (32) consisted of patients with benign pathology on evaluation. Group 2 (70) consisted of patients with no obvious pathology on investigation (fig 2). This last group was further subdivided into four subgroups. Subgroup A (37) consisted of patients who persisted with suspicious cytology or symptoms and developed malignancy diagnosed on further evaluation. The mean duration for appearance of a lesion was 5.6 months (range, 3-12). Subgroup B (four) consisted of patients in whom repeat prostate evaluation confirmed adenocarcinoma of the prostate. Subgroup C (25) comprised patients with persistent normal cytological analysis carried out at least six to eight weeks apart and who showed no evidence of malignancy on further evaluation. Subgroup D (four) comprised patients who had persistent suspicious urinary cytology with no evidence of malignancy on repeat investigation. Patients in subgroup A were subsequently found to have urological tumours (eight ureteric and 29 bladder; fig 3). Urothelial malignancy was diagnosed in 29 of these patients on further investigation after initial negative evaluation as a result of persistent positive or suspicious cytology at the time of the second investigation. Eight patients had recurrent episodes of haematuria and reevaluation showed urothelial tumours. In subgroup B, three patients had suspicious digital rectal examination results, and biopsies confirmed adenocarcinoma of the prostate. All these patients had previous evaluation for suspected carcinoma of the prostate, including biopsies with no evidence of malignancy. One patient had urinary retention and transurethral resection of the prostate showed adenocarcinoma of

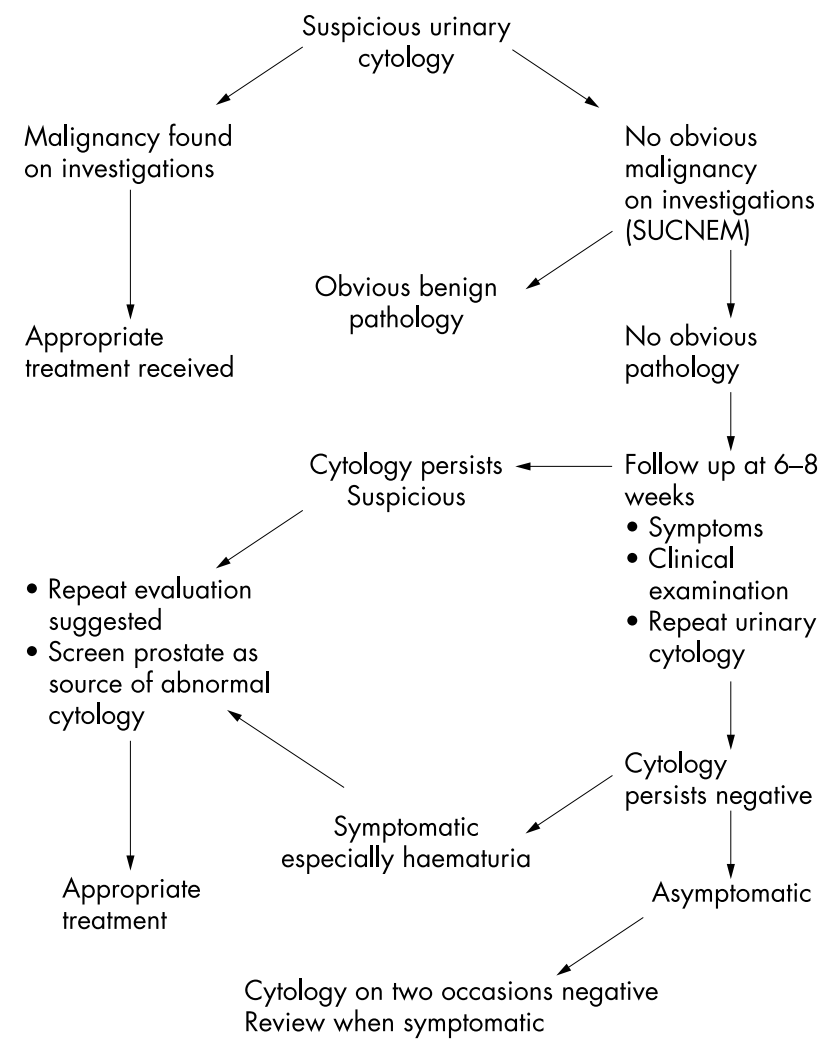

Figure 1 Algorithm for the management of suspicious urinary cytology for malignant cells in urological examination.

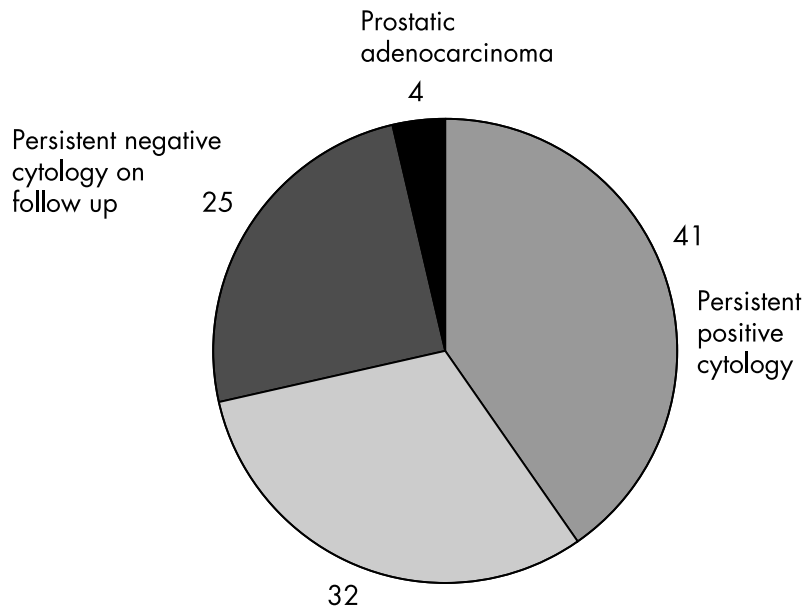

Benign pathological conditions

with negative follow up cytology

Figure 2 Diagnostic outcome of group 1 patients (32 patients with benign pathology on evaluation) and group 2 patients ( 70 patients with no obvious pathology on evaluation).

the prostate. None of the patients with benign pathology (stones, longterm catheters, pelviureteric junction obstruction, previous instrumentation) or normal cytological analysis on repeat evaluation was found to have evidence of malignancy on follow up. The presence of suspicious cells on repeat urine analysis was the only significant factor in predicting the presence of urothelial tumours $(p=0.002)$. Age, sex, and history of smoking were not significant.

\section{DISCUSSION}

In 1864, Sanders first described the presence of exfoliated neoplastic cells in the urine. ${ }^{2}$ In $1945,{ }^{1}$ its use in the diagnosis of urothelial malignancy was reported. Since that time, urine cytology has gained widespread acceptance as a useful investigation of individuals with haematuria. However, despite its extensive use in routine practice, urine cytology has several important drawbacks. Several large series have shown that urine cytology has a low sensitivity, varying between $42 \%$ and $66 \%$ depending on the population studied. Specificity rates are higher, but rarely exceed $97 \%$ in routine practice. $^{3-8}$

Unlike other forms of cytological assessment, such as breast cytology, urine cytology does not target an abnormal area but relies on neoplastic cells being shed in the urine. It is well recognised that high grade urothelial tumours, including carcinoma in situ, are more likely to shed abnormal cells into the urine. These cells are more atypical, permitting easier and more confident recognition by the reporting cytologist. It is not surprising then that sensitivity rates are higher for tumours of higher grade. The corollary is that low grade tumours are less likely to shed cells into the urine. The cells that are shed vary little from normal urothelial cells, apart from a tendency to be shed in clusters rather than as single cells. Interpretation of these subtle changes is very difficult. Additional problems in urine cytology are that stones, infections, urinary tract instrumentation, and chemotherapy/radiotherapy can all cause changes in the urine cytology picture that mimic malignancy, leading to an erroneous diagnosis by the unwary. ${ }^{9-11}$

Urine cytology specimens reported as being suspicious of malignancy require further clinical evaluation of the patient by the attending urologist. This can cause undue anxiety in the patient who is worried about the prospect of undiagnosed malignancy and who has to face a series of often 


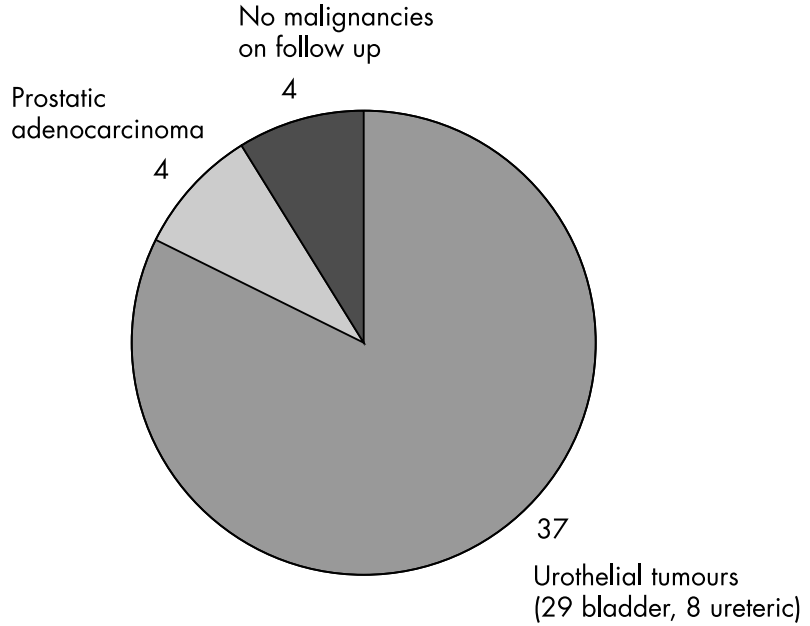

Figure 3 Final outcome of patients with persistent abnormalities on follow up. Thirty seven patients had urothelial tumours, four had prostatic adenocarcinoma, and four had no evidence of malignancy on further evaluation.

uncomfortable and unpleasant investigations. The urologist may be faced with the situation where all other initial investigations are negative apart from urine cytology, and it is often unclear how to proceed with follow up in the absence of specific guidelines. Most studies that have evaluated the outcome of patients who have been reported as having atypical urine cytology show that less that 50\% of these individuals are found to have malignancy. This suggests that only half of those with atypical urine cytology need careful follow up, and that currently a large number of patients are subjected to unnecessary investigations, with resultant wastage of valuable resources and time. The aim of our study was to try to assess whether there were specific criteria that could determine those patients who need to be subjected to careful follow up and those patients who could safely be discharged.

\section{"The injudicious use of urine cytology in individuals who have an obvious cause of haematuria, such as urinary tract stones, is to be avoided because it may lead to suspicious results being generated"}

Thirty one percent of our patients with an initial suspicious urinary cytology result and negative initial evaluation were found to have a benign disease that could explain their abnormal result. None of this group had abnormal cytology results on repeat follow up and all of the patients are now asymptomatic. This group highlights the problem of benign pathology causing significant atypia in voided urothelial cells. It also emphasises the need to provide accurate and complete clinical details to the pathology department when submitting the specimen. Urine cytology specimens are high volume specimens. Frequently, the request form arrives with no clinical details, which can lead to misinterpretation of the changes by the reporting cytopathologist. This problem is compounded by increasing workload pressures on pathology laboratories, which prevents follow up of each abnormal urine specimen. The injudicious use of urine cytology in individuals who have an obvious cause of haematuria, such as urinary tract stones, is to be avoided because it may lead to suspicious results being generated. This emphasises the need for urologists to have strict criteria governing which individuals should be subjected to urine cytology to avoid this happening.

\section{Take home messages}

- Patients with suspicious urinary cytology with negative initial evaluation should have repeated urinary cytology analysis carried out at least six to eight weeks later

- Asymptomatic patients or patients with obvious benign pathology do not require repeat evaluation

- Those with persistent positive cytology or recurrent haematuria need further evaluation and follow up and careful evaluation of the prostate should be carried out in these patients

Four patients in our study group had persistently atypical cells in their urine despite initial normal evaluation. All of these patients were found to have abnormal prostates on review examination, and were confirmed to have prostate adenocarcinoma on histology. In our present series, all patients showed high grade adenocarcinoma (Gleason score $>7$ ) on histology. This is similar to that reported previously by Rupp et al. ${ }^{12}$ Several reports have described the cytological features of malignant cells from prostatic carcinoma within voided urine samples. Krishnan and colleagues ${ }^{13}$ described the cells as having oval nuclei with smooth borders, powdery evenly distributed nuclear chromatin, large prominent nucleoli, and lacking appreciable pleomorphism. However, the recognition of these subtle differences is difficult, and distinguishing these cells from atypical cells of urothelial type may be almost impossible except in rare circumstances. In male patients with persistently atypical cells in their urine, careful assessment of the prostate is recommended, particularly when there is no evidence of malignancy on initial evaluation. In selected cases, there may also be a role for immunohistochemical staining for prostate specific antigen on the urine sample to help identify the profile of the atypical cells. ${ }^{14}{ }^{15}$

Forty one $(40 \%)$ of our patients had persistent haematuria or persistent atypical cells in their urine despite a negative evaluation for malignancy at the initial diagnostic investigation. Thirty seven of these patients were subsequently found to have a urothelial malignancy on repeat evaluation. Eight of these occurred in the ureter and 29 in the bladder. The mean duration for the appearance of these lesions was 5.6 months (range, 3-12). The reasons why the bladder tumours were not readily apparent at initial assessment are unclear. It may be that the tumours were of small size, were in areas of the bladder not readily assessable with a flexible cystoscope, or that the operator was inexperienced or under time pressure because of a high throughput of patients. Our study shows that patients who have persistently abnormal urine cytology should be assessed very carefully, preferably with rigid cystoscopy, and by an experienced urologist before the bladder and upper tracts can be said to be normal.

There still remains a small group of four patients (3\%) within our study who showed suspicious cytology despite careful follow up. As in other areas of cytology, there will always be a small false positive rate even in the most expert centres. However, it is probably worthwhile reviewing the specimens in this group and considering seeking an expert opinion. It is difficult to recommend how long these patients should be followed for. Because most of the tumours in our series presented in the first 12 months after an abnormal cytology result, it would seem appropriate to review them regularly for 12-18 months and then to consider discharging them back to primary care 


\section{CONCLUSIONS}

Patients with suspicious urine cytology with a negative initial evaluation should have a repeat urine cytology examination six to eight weeks later. Asymptomatic patients with negative repeat cytology and patients with an obvious benign pathology that can explain their abnormal result do not require repeat evaluation. Patients with persistent positive cytology or recurrent haematuria need further careful evaluation, including assessment of the prostate. Most patients in this group will subsequently be found to have an underlying malignancy.

\section{Authors' affiliations}

G Nabi, Academic Urology Unit, Department of Surgery, University of

Aberdeen, Aberdeen AB25 2ZD, UK

D Greene, Department of Urology, Royal Sunderland Hospital, Sunderland SR4 7TP, UK

M O Donnel, Department of Pathology, Royal Sunderland Hospital

\section{REFERENCES}

1 Papanicolaou GN, Marshal VF. Urine sediment smears: a diagnostic procedure in cancers of urinary tract. Science 1945;101:519-20.

2 Sanders WR. Cancer of the bladder fragments forming urethral plugs discharged in urine: concentric colloid bodies. Edinburgh Journal of Medicine 1864; 10:273-4.
3 Nabi G, Greene DR, O Donnel Marie. How important is urinary cytology in the diagnosis of urological malignancies. Eur Urol 2003;43:432-6.

4 Chahal R, Gogoi NK, Sundaram SK. Is it necessary to perform urine cytology in screening patients with haematuria? Eur Urol 2001;39:283-6.

5 Sharma S, Zippe CD, Pandrangi L, et al. Exclusion criteria enhance the specificity and positive predictive value of NMP22 and BTA stat. J Urol 2000;162:53-7.

6 Raitanen MP, Aine RAT, Kaasinen ES, et al. Suspicious urine cytology (class III) in patients with bladder cancer: should it be considered as negative or positive? Scan J Urol Nephrol 2002;36:213-17

7 Paez A, Coba JM, Murillo N, et al. Reliability of the routine cytological diagnosis in bladder cancer. Eur Urol 1999:35:228-32.

8 Amberson JB, Laino JP. Image cytometric deoxyribonucleic acid analysis of urine specimens as an adjunct to visual cytology in the detection of urothelial cell carcinoma. J Urol 1993;149:42-5.

9 Johnston B, Morales A, Emerson L, et al. Rapid detection of bladder cancer: a comparative study of point of care tests. J Urol 1997;158:2098-101.

10 Kannan V, Gupta D. Calculus artefact. A challenge in urinary cytology. Acta Cytol 1999;43:794-800.

11 Maier U, Shank R, Neuhold N. The clinical value of urinary cytology: 12 years of experience with 615 patients. J Clin Pathol 1995:48:314-17.

12 Rupp M, O'Hara B, McCullough L, et al. Prostatic carcinoma cell in urine specimen. Cytopathology 1994;5:164-70.

13 Krishnan B, Truoug LD. Prostatic adenocarcinoma diagnosed by urinary cytology. Am J Clin Pathol 2000;113:29-34.

14 Varma VA, Fekete PS, Franks MJ, et al. Cytologic features of prostate adenocarcinoma in urine. A clinicopathological and immunocytochemical study. Diagn Cytopathol 1988;4:300-5.

15 Schnadig VJ, Adesokan A, Neal D Jr, et al. Urinary cytological findings in patients with benign and malignant adenomatous polyps of the prostatic urethra. Arch Pathol Lab Med 2000;124:1047-52.

\section{Teaser}

\section{POPULAR PATHOLOGY REQUISITIONS: THE HIDDEN MEANING}

(1) For urgent processing: pleeeaase, the boss is breathing down my neck, and I haven't the faintest clue what this is, save me!

(2) Diffuse mildly enlarged thyroid: I didn't feel it, the boss did.

(3) Ill defined nodularity in the upper, outer quadrant of the right breast for FNAC: I saw you joblessly loitering in the canteen, so I'm sending some business your way; enjoy the hide and seek!

(4) 22 year old woman with vaginal bleeding every 28 days, to rule out endometrial pathology: now, I am jobless.

(5) FNAC benign, frozen section to rule out malignancy: buddy, I don't trust you.
(6) Please issue duplicate slides for further management: I still don't trust you.

(7) $2500 \mathrm{ml}$ of urine from a dipsomaniac, to look for malignant cells: I didn't know how to discard it.

(8) $0.5 \times 0.5 \mathrm{~cm}$ axillary node for FNAC, patient is HIV positive: I don't have the nerve to biopsy it.

(9) Please look for Helicobacter pylori: the consumer forum president has become flatulent; scopy's normal, tummy's no trouble; dig into that haystack, find a needle, and save us all from the ordeal.

(10) Request for a complete necropsy on a patient who died 420 days after an aspirate from a lipomatous swelling on his right little toe: we don't know why he died, but we're hoping to pin the blame on you.

T Rajalakshmi

Department of Pathology, St. John's Medical College, 428, 7th cross, 1st block, Jayanagar, Bangalore 560011 , India; rajtiru@hotmail.com 


\section{PostScript}

\section{CORRESPONDENCE}

\section{Authorship trends in the Indian Journal of Pathology and Microbiology: going the global way?}

Guidelines for authorship of biomedical journals have been available for nearly two decades now to help determine how attribution should be acknowledged. ${ }^{1}$ With an increasing number of multiauthored articles and with contributions from diverse specialties, authorship disputes will always raise their head because existing guidelines may not be followed stringently or may be misinterpreted. I analysed author numbers in the Indian Journal of Pathology and Microbiology over the past 28 years (1975-2002). The designation of various authors or their individual contributions to the authored papers was not ascertained. In total, 1861 articles comprising $1268(68.1 \%)$ original articles and $593(31.9 \%)$ case reports were studied.

The number of authors for original articles ranged from one to a maximum of 10 (mean, 3.4). The mean number of authors for each article showed a significant increase from 1.9 (SD, 1.4) in 1975 to 3.9 (SD, 1.2) in 2002. The proportion of original articles with single authors declined from 50\% in 1975 to none in 2002. Original articles with five or more authors also showed a sharp rise from 5\% in 1975 to more than one fourth $(26.1 \%)$ of all articles in 2002.

The number of authors for 593 published case reports ranged from one to a maximum of eight (mean, 3.5). The mean number of authors for each article showed a significant increase from 2.6 (SD, 1.5) in 1975 to 4.2 (SD, 1.3) in 2002. The proportion of case reports with single authors declined from $40 \%$ in 1975 to none in 2002. There were no articles with five or more authors in 1975, whereas $40 \%$ of case reports published in 2002 were authored by five or more researchers.

The International Committee of Medical Journal Editors (Vancouver group) drew up the guidelines for authorship based on the principle that each author should be able to defend the work publicly. However, several studies have shown that these guidelines are not followed and the literature abounds with examples of irresponsible authorship. ${ }^{2-4}$ Some researchers have recommended doing away with the present designation of author and replacing it with that of a contributor. ${ }^{5}$ In India, with many researchers being unfamiliar with the Vancouver guidelines, authorship is, on many occasions, a political decision determined by the position of the author in the hierarchy ladder. Not many junior researchers have the courage to refuse polite implicit or explicit requests from their seniors for inclusion as co-authors, even if their contribution to the work was negligible. The complex factors in the authorship tangle can operate in the reverse direction also, with juniors including senior investigators as co-authors to increase the credibility of the paper and its chances of being accepted. The conferral of gift authorship also erodes the pillars of ethical attribution. All these practices violate the Vancouver guidelines and cause much anguish to those forced to include noncontributors as co-authors in their work or those who are denied authorship when it is deserved. Authors must accept responsibility for a publication when taking the credit for the same. Institutions also need to address authorship issues head on, and devise ways to deal with such disputes. This vital activity in the biomedical field should not be converted into a power game where only the mighty win.

N Kakkar

Department of Pathology, Christian Medical College and Hospital, Ludhiana 141008 , Punjab, India; n kakkar@satyam.net.in

\section{References}

1 International Committee of Medical Journal Editors. Uniform requirements for manuscripts submitted to biomedical journals. JAMA 1993;269:2282-6.

2 Bhopal R, Rankin J, McColl E, et al. The vexed question of authorship: views of researchers in a British medical faculty. BMJ 1997;314:1009-12.

3 King JT. How many neurosurgeons does it take to write a research article? Authorship proliferation in neurosurgical research. Neurosurgery 2000;47:435-40.

4 Shapiro DW, Wenger NS, Shapiro MF. The contribution of authors to multiauthored biomedical research papers. JAMA 1994;271:438-42.

5 Rennie D, Yank V, Emanuel L. When authorship fails. A proposal to make contributors accountable. JAMA 1997;278:579-85.

\section{Type A intracytoplasmic inclusions in human cowpox infection}

Replication of poxviruses results in the development of intracytoplasmic inclusions that may be visible by light microscopy. Kato et al (1959) classified these according to their morphology, staining properties, and rate of accumulation. ${ }^{1}$ Type B inclusions are basophilic and occur early in the viral replication cycle. They represent the actual site of viral replication in the cytoplasm and may be seen in all productive poxvirus infections. In contrast, type A inclusions are large, well defined, eosinophilic bodies that develop late in the viral replication cycle. They are composed entirely of a single protein species that has a molecular weight of $160 \mathrm{kDa}$, but their function is not understood. ${ }^{2}$ Type A inclusions are only associated with certain poxvirus infections so that their detection may be diagnostically useful.

We recently reported the clinical presentation of a veterinary surgeon working at a small animal practice who developed a black necrotic ulcer on his finger. ${ }^{3}$ Histopathology of a punch biopsy of the lesion unexpectedly revealed epidermal hyperplasia, neutrophil infiltration, and the presence of numerous large eosinophilic intracytoplasmic inclusions, consistent with poxvirus infection (fig 1). Based on this appearance, the differential diagnosis included cowpox (an orthopoxvirus infection), orf, and pseudocowpox (both parapoxvirus infections). In the context of frequent

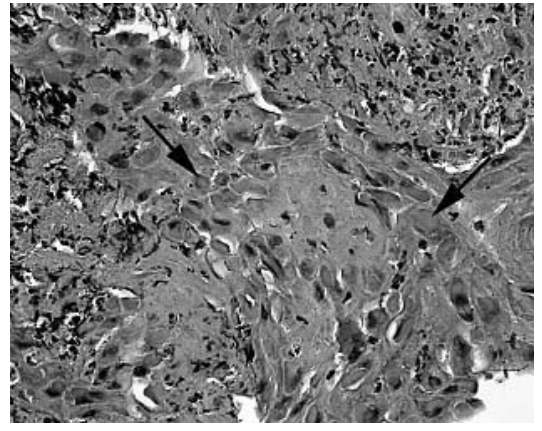

Figure 1 Histological examination of a punch biopsy of the skin ulcer stained with haematoxylin and eosin (medium power). Numerous, large, eosinophilic type A intracytoplasmic inclusions are seen (arrows).

occupational exposure to cat scratches but no contact with ungulates, cowpox was the most probable diagnosis. This diagnosis was confirmed by polymerase chain reaction and partial DNA sequencing. Human cowpox is an uncommon and probably underdiagnosed infection that occurs only in Europe. ${ }^{4}$ Despite the name, cowpox is rare in cattle. Human infections are probably most frequently acquired from infected cats shedding virus from skin lesions, which gain entry to the human skin through abrasions or scratches. ${ }^{45}$

Laboratory diagnosis of cowpox may be established by electron microscopy of vesicle fluid, by polymerase chain reaction, virus isolation, or serology. However, in our case the histopathological appearances were important in establishing a probable diagnosis of human cowpox before molecular virological confirmation at a reference laboratory. Cowpox virus is recognised as causing large intracytoplasmic inclusion bodies in infected feline tissues and in in vitro cell culture. ${ }^{4}$ However, such inclusions were not reported among the biopsied cases included in an extensive review of this disease, in which a total of 54 published and unpublished human cases were reviewed. Indeed, an extensive search of the literature found no previous reports of such inclusions being seen in biopsied human cowpox lesions Histopathologists should be aware that such a histological appearance in an unusual vesicular, pustular, or ulcerated skin lesion obtained from a patient with a history of contact with domestic cats probably results from cowpox infection.

S D Lawn

Department of Cellular and Molecular Medicine Infectious Diseases, St George's Hospital Medical School, London SW17 ORE, UK stevelawn@yahoo.co.uk

S Holwill

Department of Histopathology, St George's Hospitc Medical School

\section{References}

1 Kato S, Takahashi M, Kameyama S, et al. A study of the morphological and cyto-immunological relationship between the inclusions of vaccinia 
rabbitpox, vaccinia (variola origin) and vaccinia IHD and a consideration of the term "Guarnieri body". Biken's Journal 1959;2:343-63.

2 Patel DD, Pickup DJ, Joklik WK. Isolation of cowpox virus A-type inclusions and characterization of their major protein component. Virology 1986;149:174-89.

3 Lawn SD, Planche T, Riley P, et al. A black necrotic ulcer. Lancet 2003;361:1518.

4 Baxby D, Bennett M, Getty B. Human cowpox 1969-93: a review based on 54 cases. Br J Dermatol 1994;131:598-607.

5 Willemse A, Egberink HF. Transmission of cowpox virus infection from domestic cat to man Lancet 1985;i: 1515

\section{Omental actinomycosis presenting with right lower quadrant abdominal pain}

A previously healthy 21 year old man presented with a two day history of worsening right lower quadrant abdominal pain. On examination he was found to have a fever $\left(39^{\circ} \mathrm{C}\right)$ and his pulse rate was 110 beats/ minute. There was tenderness with muscle guarding in the right lower quadrant, which worsened after a period of observation. Basic haematology and biochemistry tests were normal. Plain $x$ rays of the chest and abdomen were also normal.

A diagnosis of acute appendicitis was made and the patient was transferred for emergency appendicectomy. The appendix proved to be macroscopically normal but a hard and fairly mobile mass was discovered in the right upper quadrant. We decided to extend the original Lantz incision transversely to improve access. The mass was arising from the right side of the greater omentum. It was loosely adherent to the proximal third of the transverse colon and the overlying peritoneum. The other viscera were normal. The mass was freed and excised with a $1 \mathrm{~cm}$ margin of macroscopically unaffected omentum. It was round in shape, $7 \mathrm{~cm}$ in diameter, and light brown in colour with an uneven surface.

Histological examination using haematoxylin and eosin staining confirmed that the specimen was a non-caseating granulomatous mass featuring eosinophilic granules. A Gram stained smear prepared from the lesion revealed sulfur granules characteristic of actinomycosis, made up of colonies of Gram positive actinomycetes. Therefore, the mass was an omental actinomycoma.

The patient made an uneventful postoperative recovery. We treated him with intravenous crystalline penicillin, 4MU six hourly for two weeks. Before discharge we investigated the patient's gastrointestinal tract with a barium meal with follow through examination to rule out the possibility of an underlying predisposing condition, such as inflammatory bowel disease or an intraluminal lesion. He was discharged on oral amoxicillin, $500 \mathrm{mg}$ eight hourly for a total of four months. We reviewed him six and then 12 months after surgery. On both occasions his white blood cell count, erythrocyte sedimentation rate, $\mathrm{C}$ reactive protein, and computed tomography scan of the abdomen and pelvis were completely normal.

Abdominal actinomycosis is an unusual condition, which tends to be a disease of insidious onset and vague symptoms. ${ }^{1}$ Recognised causal associations include a history of appendicitis, ${ }^{2}$ diverticulitis, ${ }^{3}$ inflammatory bowel disease, intrauterine contraceptive device use, or open and endoscopic surgery. However, we

found no such association in our patient. Preoperative diagnosis is difficult and requires a high index of suspicion. When actinomycosis is suspected, computed tomography guided aspiration, with or without core biopsy of suspicious lesions, is a useful investigation. ${ }^{5}$ This may reduce the number of patients undergoing radical surgery for this benign disease. The role of surgery should be limited to cautious resection of the lesions with delivery of suitable specimens for histology and microbiology.

The organisms tend to be sensitive to cephalosporins, tetracyclines, and macrolides. The duration of treatment is a controversial issue, but long courses are recommended. ${ }^{1}$ The prognosis for patients suffering from abdominal actinomycosis is good provided that adequate antibiotic cover is instituted at an early stage.

\section{J Abela \\ Lister Department of Surgery, Glasgow Royal Infirmary, Glasgow G4 OSF, UK jeabela@hotmail.com}

J Sciberras, M Meilak, A G Felice Department of Surgery, St Luke's Hospital MSD09,

Malta

J Degaetano

Department of Pathology, St Luke's Hospital

\section{References}

1 Shaal KP. Actinomycoses, actinobacillus and related diseases. In: Collier L, Balows A, Sussman $M$, eds. Topley and Wilson's microbiology and microbial infections, Vol. 3, 9th ed. London: Arnold, 1998:777-98.

2 Schmidt P, Koltai JL, Weltzien A. Actinomycosis of the appendix in childhood. Pediatr Surg Int 1999; 15:63-5.

3 Samuel I, Dixon MF, Benson EA. Actinomycosis complicating chronic diverticulitis of the sigmoid colon: a missed association? Postgrad Med J 1992;68:57-8.

4 Asuncion CM, Cinti DC, Hawkins HB. Abdominal manifestations of actinomycosis in IUD users. J Clin Gastroenterol 1984;6:343-8.

5 Pombo F, Rodriguez E, Martin R, et al. CT-guided core-needle biopsy in omental pathology. Acta Radiol 1997;38:978-81.

\section{Incidental freezing artefacts in sentinel lymph node biopsies masquerading as lymphangiography artefacts}

Sentinel lymph node (SLN) biopsy is a widely used staging procedure. As recently reviewed, possible problems with microscopic interpretation include false positive immunohistochemical staining and benign lesions mimicking metastasis. ${ }^{1}$ To my knowledge, no histological artefacts, especially no lymphangiography artefacts, have been attributed to this procedure. I recently encountered an artefact that at first glance seemed to be a lymphangiography artefact.

SLN biopsy is used in our hospital for the staging of breast cancer and was introduced in July 2001. The details of our procedure are as follows. The radioactive tracer is injected intracutaneously or subcutaneously in the affected quadrant of the breast 24 hours before surgery. On the morning of the operation, patent blue dye is injected intracutaneously or subcutaneously in the affected quadrant of the breast. SLN biopsies are fixed in formalin and stored in the operating theatre for 24 hours, after which time, the

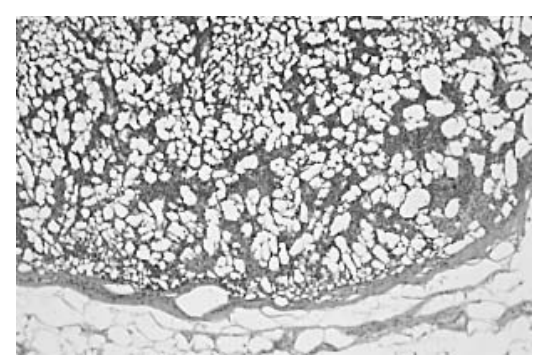

Figure 1 Incidental freezing artefacts in a sentinel lymph node. Lymph node showing empty holes with no reactive changes.

amount of radioactivity is low enough for the specimens to be transported by public highway with no special measures or a licence.

In March 2003, we received two SLN biopsies on the same day, both with artefacts. Throughout the lymph nodes there were empty holes with no reactive changes (fig l). A similar artefact was noted in the tumour of one of the lumpectomy specimens, making grading of this tumour impossible. All tissues seemed to be adequately fixed in formalin and paraffin wax embedded. No artefacts were noted in other specimens that were processed on the same day. A link was made with the SLN procedure and the artefacts were interpreted as possible lymphangiography artefacts. The departments of surgery and nuclear medicine were contacted to ask whether there had been any changes in the SLN procedure. Both of these departments denied changes to their procedures; specifically, neither of the two tracers was injected in or near the tumour and there was no change in the composition of the tracers. The SLNS taken in 2002 were reviewed and it was noted that the first SLN with the same artefact was taken in the last week of December and the same artefact was also seen in SLNs from January and February 2003. However, the lymph nodes were assessed by different pathologists and the artefacts were not interpreted as related to the SLN procedure.

The department of surgery was again contacted and one of the nurses from the operating theatre mentioned that the SLN biopsies were stored in a refrigerator until transportation. This refrigerator had been switched on after reorganisation of the operating theatre in December 2002, but before that date it had been switched off. On several occasions she had noticed that tissues stored in the refrigerator were frozen and for this reason the refrigerator had been serviced twice, although no improvement was seen. This problem had not been reported to our laboratory and we had never noticed that the SLN specimens were frozen on arrival. We concluded that the artefacts in the SLNs were freezing artefacts and the refrigerator was switched off. Since then the artefact has disappeared.

P J Westenend

Pathologisch Laboratorium voor Dordrecht eo, Laan van Londen 1800, Dordrecht, 3317 DA, The Netherlands; pwestenend@paldordt.com

\section{Reference}

1 Hoda SA, Chiu A, Resetkova E, et al. Pathological examination of sentinel lymph node in breast cancer: potential problems and possible solutions. Microsc Res Tech 2002;59:85-91. 


\section{Can routine laboratory data guide empirical prescribing?}

Smellie and colleagues have noted large and significant differences in rates of submission of samples for microbiological testing between practices in the south west and north east regions. ${ }^{1}$ They think that these data indicate that some practices are more selective in the use of the laboratory and speculate that routine laboratory generated antibiotic surveillance data will thus tend to include more complicated cases and overestimate antibiotic resistance. The authors therefore question the validity of using routine laboratory data on antibiotic resistance for primary care based therapeutic guidelines for empirical prescribing, and recommend an enhanced surveillance programme with a standardised approach to testing as a better way of informing such guidelines. We have recently completed such a programme for urinary tract infection (UTI) and can therefore comment on these suggestions.

The susceptibility data for uropathogens recovered from routine urine samples received from the 80 practices served by Cambridge Microbiology and Public Health Laboratory were compared with the results from a sentinel group of five practices in the same locality, which agreed to submit urine samples on all patients presenting with a clinical diagnosis of UTI within a three month period during 2002

In total, 967 urine samples were received from the sentinel practice group and 18892 from the general practice group. Bacteria were recovered from 269 and 4449 samples from the two groups, respectively. Overall, $89 \%$ were Gram negative bacilli and the numbers of these were large enough to permit meaningful comparison and statistical evaluation. There were no significant differences in recovery of any species between the two groups, indicating that, in contrast to the assertion of Smellie et al, any bias by general practitioners to send in samples with complicated infections involving more resistant organisms, such as Pseudomonas spp, did not result in overestimating the extent of antibiotic resistance in our population.

Resistance rates to cefalexin, norfloxacin, and gentamicin were marginally lower $2.5 \%$ $v 5.2 \%, 2.1 \% \vee 4.4 \%$, and $0.8 \% \vee v 1.3 \%$, respectively) and resistance to ampicillin, trimethoprim, and co-amoxiclav was slightly higher $(46.0 \% v 45.1 \%, 22.8 \% v 19.8 \%, 18.1 \%$ $v 11.7 \%$, respectively) in the sentinel practice group than in the general practice group. However, only the resistance rate for coamoxiclav was significantly different $(\mathrm{p}=$ 0.03 by two tailed $\chi^{2}$ test)

Two other studies of enhanced surveillance programmes have been published, also investigating UTI. Baerheim et al reported a study on female patients with UTI in general practice in Norway, comparing resistance rates for bacteria recovered from unselected (sentinel) patients with those from whom urine was sent routinely, using a panel of six antibiotics. ${ }^{2}$ Uropathogens from the sentinel group were $3.4-8.4 \%$ less resistant to the antibiotics tested, but the results were significant only for nitrofurantoin $3.0 \%$ resistant in sentinel patients compared with 9.7\% resistant in routine patients).

Richards reported a study similar to our own over a 12 month period involving three sentinel general practices in Norfolk, UK. Sentinel practice resistance rates were the same, or very slightly higher for co-amoxiclav, ciprofloxacin, cefradine, and gentamicin and slightly lower for ampicillin, cefuroxime, and trimethoprim. The difference was largest with trimethoprim (18\% resistant in sentinel practice patients versus $22 \%$ resistant in all other practices), and only this difference achieved significance. We did not duplicate their findings; indeed, the pattern for lesser or greater resistance in the sentinel practice group versus all general practice was reversed in our study.

There is no consistency in antibiotics showing greater or lesser resistance rates in sentinel practice patients in these studies. However, a common feature of all three is that the differences in resistance rates for sentinel practices compared with overall reported resistance rates are small, and none would justify a change in recommendations for empirical prescribing for UTI in general practice. We conclude that there is good evidence that susceptibility data derived from routine urine samples received by the laboratory provide reliable information for formulating empirical prescribing guidelines for urinary tract infections in domiciliary practice. We suspect that this also applies to specimens from other sites, but this requires further study.

Health Protection Agency, Clinical Microbiology and Public Health Laboratory, Addenbrooke's Hospital, Cambridge CB2 2QW, UK; hugo.ludlam@ addenbrookes.nhs.uk

O Sule

Clinical Microbiology Laboratory, Royal Free Hospital, London NW32Q9, UK

M Knapton

Cambridge City Primary Care Trust, Heron Court, Ida Darwin, Fulbourn, Cambridgeshire CB1 5EE, UK

I Abubakar

Communicable Disease Surveillance Centre Eastern, IPH, University Forvie Site, Robinson Way, Cambridge CB2 2SR, UK

\section{References}

1 Smellie WSA, Clark G, McNulty CAM Inequalities of primary care microbiology testing between hospital catchment areas. J Clin Pathol 2003;56:933-6.

2 Baerheim A, Digranes A, Steinar H. Are resistance patterns in uropathogens published by microbiology laboratories valid for general practice? APMIS 1999; 107:676-80

3 Richards J. Monitoring antibiotic resistance in urinary isolates from the community-a spotter practice model. Communicable Disease and Public Health 2002;5:226-9.

\section{BOOK REVIEW}

\section{Surgical pathology dissection. An illustrated guide, 2nd ed}

Westra WH, Hruban RH, Phelps TH, Isaacson C. (£56.00.) Springer, 2003. ISBN 0387 955593.

Because I am particularly obsessed with standardisation of the grossing of specimens, reviewing a book devoted entirely to this extremely important aspect of surgical pathology was something I did with relish.

As is set out in the foreword, the grossing guidelines contained in the book conform, in the main, with the recommendations of the College of Pathologists and the Association of Directors of Anatomic and Surgical Pathology, although the contributors are from one institution.

The style of the book is very simple and the reader is "talked" through the dissection. There are line drawings of the specimens and where sections of the specimen should be taken from. Although in black and white, this does not detract from the essence of the book. As such, it is an excellent instruction manual that should form the basis of every cut up manual anywhere in the world. Although there are regional variations and personal quirks, there is only one way to gross a specimen properly and that is the right way. This book will point anyone who is at the cut up bench in the right direction.

I thoroughly recommend this book to all registrars, pathologists, and pathologist's assistants.

R Chetty

\section{CALENDAR OF EVENTS}

Full details of events to be included should be sent to Maggie Butler, Technical Editor JCP. The Cedars, 36 Queen Street, Castle Hedingham, Essex CO9 3HA,UK; email: maggie.butler2@ btopenworld.com

\section{Practical Pulmonary Pathology}

27-30 July, 2004, Brompton Hospital, London, UK

Further details: Professor B Corrin, Brompton Hospital, London SW3 6NP, UK (Tel: +44 (0)20 7351 8420; Fax: +44 (0)20 7351 8293; Email: b.corrin@ic.ac.uk)

\section{ACP Management Course for Pathologists, 2004}

8-10 September 2004, Hardwick Hall Hotel, Sedgefield, County Durham, UK

Further details: V Wood, ACP Central Office, 189 Dyke Road, Hove, East Sussex BN3 1TL, UK. (Tel: +44 (0) 1273 775700; Fax: +44 (0) 1273 773303; Email: valerie@pathologists. org.uk

\section{Asian Pacific Association for study of the Liver Biennial Conference}

11-15 December 2004, New Delhi, India

Further details: Dr V Malhotra (General Secretary) or Dr P Sakhuja (Treasurer and Pathology Coordinator), Room 325, Academic Block, Department of Pathology, GB Pant Hospital, New Delhi 110002, India. (Tel: +9l 11 23237455; Email: welcome@apaslindia2004. com; Website: www.apaslindia2004.com)

\section{CORRECTION}

Suspicious urinary cytology with negative evaluation for malignancy in the diagnostic evaluation of haematuria: how to follow up? G Nabi, D Greene, M O O Donnel J Clin Pathol 2004:57:365-8. The third author's name was incorrectly spelt: it should have been M O O'Donnell. In addition, this author would like it to be known that she did not see the proofs before the paper was published. 